\title{
El fútbol como herramienta pedagógica en la enseñanza de dirección de empresas
}

\section{Óscar Gutiérrez Aragón}

Escola Universitària Mediterrani. Universitat de Girona. España. oscar.gutierrez@mediterrani.com

\section{Carmen María Vizoso Gómez}

Universidad de León. España.

cvizg@unileon.es

\section{Joan Francesc Fondevila Gascón Gaspar Berbel Giménez}

Escola Universitària Mediterrani. Universitat de Girona. España. jf.fondevila@mediterrani.com gaspar.berbel@mediterrani.com

\section{Resumen}

La implantación del Espacio Europeo de Educación Superior ha puesto de relevancia la necesidad de que el profesorado universitario desarrolle y adopte nuevas formas de docencia que permitan a los alumnos acceder al aprendizaje de las asignaturas desde una nueva perspectiva didáctica. El objetivo principal de este trabajo es analizar los efectos de la puesta en práctica de proyectos de innovación docente en el rendimiento académico en una muestra formada por 1.546 estudiantes de tres universidades y cuatro grados diferentes. Para ello se ha comparado el rendimiento de un grupo experimental, que realizó una actividad práctica que relacionaba el triunfo de la selección española de fútbol en el Mundial de Sudáfrica del año 2010 con la forma en que se ejecutan las principales funciones de la dirección de una empresa, con el rendimiento de un grupo control, que basó el mismo aprendizaje en la tradicional clase magistral. Los resultados obtenidos muestran que el grupo experimental alcanzó un rendimiento académico superior al del grupo control. Por lo tanto, se concluye que el desarrollo y la implantación de este proyecto de innovación docente mejora el rendimiento académico en los estudiantes universitarios.

Palabras clave: innovación pedagógica; rendimiento; estrategias de aprendizaje activo; formación empresarial; ciencias económicas 


\section{Resum. El futbol com a eina pedagògica en l'ensenyament de direcció d'empreses}

La implantació de l'Espai Europeu d'Educació Superior ha posat de rellevància la necessitat que el professorat universitari desenvolupi i adopti noves formes de docència que permetin que els alumnes accedeixin a l'aprenentatge de les assignatures des d'una nova perspectiva didàctica. L'objectiu principal d'aquest treball és analitzar els efectes de projectes d'innovació docent en el rendiment acadèmic en una mostra formada per 1.546 estudiants de tres universitats i quatre graus diferents. Per aquest motiu s'ha comparat el rendiment d'un grup experimental, que va realitzar una activitat pràctica que relacionava el triomf de la selecció espanyola de futbol al Mundial de Sud-àfrica de l'any $2010 \mathrm{amb}$ la manera com s'executen les principals funcions de la direcció d'una empresa, amb el rendiment d'un grup control, que va basar el mateix aprenentatge en la tradicional classe magistral. Els resultats obtinguts mostren que el grup experimental va aconseguir un rendiment acadèmic superior al del grup control. Per tant, es conclou que el desenvolupament i la implantació de projectes d'innovació docent realitzats en les aules millora el rendiment acadèmic en els estudiants universitaris.

Paraules clau: innovació pedagògica; rendiment; estratègies d'aprenentatge actiu; formació empresarial; ciències econòmiques

\section{Abstract. Football as a pedagogical tool in business management education}

Following the implementation of the European Higher Education Area, university teachers have had to develop and apply new teaching methods in their educational practice that enable students to learn from a more didactic approach. The main objective of this paper is to analyse the effects of the implementation of teaching innovation projects on academic performance in a sample of 1,546 students from three universities and four different degree programmes. To this end, we studied the performance of an experimental group that carried out a practical activity in which they had to relate the victory of the Spanish national soccer team in the 2010 World Cup in South Africa with how the main functions of a company's management are executed. The experimental group was compared to a control group that studied the same topic in a traditional master class. The results show that the academic performance of the experimental group was superior to that of the control group. Therefore, it is concluded that the development and implementation of teacher innovation projects carried out in the classroom improve the academic performance of university students.

Keywords: educational innovation; achievement; active learning strategies; management education; economics

\section{Sumario}

1. Introducción

2. Metodología

3. Resultados
4. Discusión y conclusiones

Referencias bibliográficas 


\section{Introducción}

El reto que ha supuesto la convergencia europea en la educación superior ha sido el origen del desarrollo de un gran número de actuaciones docentes que tienden a facilitar el aprendizaje de los estudiantes y a mejorar la calidad educativa de las asignaturas en la universidad. Este cambio trajo consigo una reforma en los estudios superiores de la mayor parte de las universidades españolas, planteando el desafío de intentar transformar unas prácticas pedagógicas centradas en la enseñanza del profesorado en otro tipo de enseñanza basada en competencias, genéricas y específicas, que buscara el desarrollo de capacidades, habilidades y valores de los estudiantes mediante metodologías docentes diferentes (Salas, Sánchez y Rodríguez, 2012; Poblete, Bezanilla, Fernández y Campo, 2016).

En este contexto de renovación docente se ha puesto de relevancia la necesidad de que el profesorado (especialmente el universitario), en un proceso dinámico y permanente de reinterpretación de creencias, valores y experiencias derivados de los nuevos marcos contemporáneos de relaciones, desarrolle y adopte modernas e inéditas estrategias docentes, promoviendo el pensamiento creativo y crítico de los contenidos curriculares y culturales (Correa et al., 2015). Así, los alumnos accederán al aprendizaje desde una nueva perspectiva didáctica, avanzando hacia una formación que supere la dicotomía clásica entre teoría y práctica y asegurando la asimilación de unos conocimientos y de unas habilidades complejos que son necesarios para convivir y relacionarse con el entorno (Alliaud, 2015).

El marco estructural actual, en el que el estudiante se sitúa en el centro del proceso de enseñanza y aprendizaje, viene ligado a un cambio metodológico que potencia su papel activo como constructor de su proceso de aprendizaje, así como su iniciativa y su pensamiento crítico (Esteve, 2009; Pérez, 2014). Este entorno favorece la aparición de numerosas iniciativas de innovación docente emanadas de la reorganización conceptual de los sistemas educativos, de la necesidad de adecuar la enseñanza a la mejora de calidad del sistema universitario (Jano y Ortiz, 2007) y de las oportunidades de equidad y eficiencia que las nuevas tecnologías ofrecen para transmitir conocimientos y reestructurar los modos de ejercer la educación (Bolívar, Domingo y Pérez-García, 2014; Castañeda, Esteve y Adell, 2018; UNESCO, 2018).

Estas necesidades de renovación docente inducen la implementación de métodos innovadores que faciliten el aprendizaje, la autonomía intelectual, la creatividad, la colaboración y la predisposición de los estudiantes para formarse (Alonso et al., 2007; Monreal y Ruiz, 2010), alejándose de una docencia basada en la clase magistral y en el antiguo esquema de enseñanza-aprendizaje que sobrevaloraba la habilidad memorística, causando, en ocasiones, una falta de interés del alumnado hacía la formación y los conocimientos que debía adquirir (De Arriba, 2014; Fidalgo, 2011).

La colaboración en el campo de la innovación docente, fomentando el uso de diversas plataformas tecnológicas, métodos de enseñanza y formas de eva- 
luación, reconocimiento y certificación, debe conducir a un análisis colectivo que abra espacios para el intercambio, el diálogo reflexivo y la planificación conjunta de este tipo de experiencias (Brunner et al., 2019; Perrenoud, 2001; Peters, 2010), contribuyendo a formar profesionales comprometidos y competentes que hagan que la enseñanza favorezca el aprendizaje activo y que este conduzca a la adquisición de conocimientos (Fidalgo, 2011). La innovación docente no se ha de limitar a una cuestión sobre el uso de diferentes metodologías, sino que debe ser además un proceso personal sobre cómo entender la educación (González y Barba, 2016) en el que lo importante no es lo que se sabe, sino lo que se hace con lo que se sabe (Wagner, 2012).

Las instituciones de educación superior no solo han de ser transmisoras de conocimiento, sino también generadoras de él (Méndez, 2018), por lo que su profesorado, en un ejercicio de transformación personal llevado a cabo mediante procesos de reflexión docente individuales o compartidos (González y Barba, 2014; Torrado y Figuera, 2019), debe preocuparse por encontrar cualquier nuevo medio, recurso o metodología de enseñanza que permita la mejora de sus conocimientos, capacidades, actitudes y competencias en temas pedagógicos (Fernández-Ferrer y Forés-Miravalles, 2018), con el fin de formar personas competentes capaces de liderar en el futuro el progreso intelectual, económico, industrial y cultural de la sociedad (Fonseca y Rodríguez, 2009), incluyendo el acercamiento a la competitiva realidad profesional actual (Pérez, 2014).

En la enseñanza de asignaturas del área de Organización de Empresas, el objetivo de las innovaciones docentes debe ser fomentar el acercamiento de los alumnos a la realidad económica y empresarial que conforma el escenario profesional al que se van a tener que incorporar al completar sus estudios (Bustinza, Tamayo, Gutiérrez y Orozco, 2010). Los profesores de este área, para aproximar a los alumnos a una realidad social flexible y creativa, en constante cambio, determinada por las exigencias y las oportunidades de la era digital y el actual funcionamiento empresarial, deben mantenerse continuamente en formación, no solo en lo relativo a su disciplina, sino también en el uso de las nuevas metodologías, de forma que puedan guiar a los alumnos a formularse las preguntas correctas y llegar a las respuestas acertadas (De Arriba, 2014).

Así, por ejemplo, una de estas metodologías, de sencilla implantación y habituales buenos resultados académicos, es el análisis de noticias económicas y empresariales, ya que su actualidad e interés implícito ayudan a asentar los conocimientos teóricos y su aplicación a problemas concretos, así como el fomento de la capacidad de razonamiento y discusión (Iglesias, Toscano y Román, 2012). En este ámbito, la utilización de casos o temas deportivos como material para la enseñanza de economía y administración de empresas suele producir buenos resultados (García, Fernández-Gavira, Sánchez y Grimaldi, 2017), debido al mayor interés y conocimiento por parte del alumnado sobre este tipo de noticias que por las meramente económicas. Obviamente, la metodología empleada en este tipo de actividades en el aula está vinculada 
con la teoría de juegos, rama de la ciencia matemática de aplicación en economía que trata de comprender la conducta humana frente a la toma de decisiones, sus estrategias y expectativas (Nash, 1950), al buscar la interactuación de los estudiantes con el tema propuesto, para, posteriormente, analizarlo desde un enfoque doctrinario, contrastando las decisiones tomadas por los alumnos con las predicciones teóricas (Jiménez y Montijano, 2006).

En las últimas décadas, la ciencia económica se ha extendido a través de muchos campos (periodismo, sociología, derecho, política, historia, etc.) y ha acabado alcanzando al análisis de deportes, más concretamente, al relativo al fútbol (Anderson y Sally, 2013; Kuper y Szymanski, 2012; Palacios, 2014a). Si se admite que en el campo económico-empresarial la obtención de datos y su evaluación pueden aportar ventajas competitivas, el deporte tiene una condición positiva en este sentido, ya que datos difícilmente observables en otras situaciones lo son en el deporte, pudiendo aplicarles de forma directa un razonamiento económico y un análisis estadístico y econométrico (Goddard y Dobson, 2011; Lewis, 2003; Palacios, 2014b). En lo referente a la innovación docente, el análisis de casos relacionados con el fútbol puede ser interesante en el aprendizaje sobre microeconomía, finanzas, gestión y organización de empresas, economía experimental, economía del comportamiento y teoría de juegos, así como para probar teorías económicas y documentar el comportamiento humano (Palacios, 2014a; Sumpter, 2016). La utilización del mayor de los éxitos deportivos del fútbol español, su triunfo en el Mundial celebrado en Sudáfrica en 2010, como herramienta de aprendizaje en el aula universitaria resulta apropiada por su significación social y permanencia de recuerdo, y más al considerar que aquella selección de jugadores se identificó con un modelo basado en la gestión del talento, el trabajo en equipo y el liderazgo, que descubría valores empresariales como la gestión coherente, el compromiso, la orientación a resultados, el objetivo común, la apuesta por la meritocracia y los procesos más funcionales y menos jerárquicos (Cubeiro y Gallardo, 2012; Gutiérrez-Aragón, 2013; Viaña, 2013).

\section{Metodología}

La metodología empleada es de tipo cuantitativo. Se aplicó un análisis estadístico para analizar la asociación entre la variable exposición y la variable resultado en 1.546 alumnos de doce grupos de cuatro grados diferentes en cuatro asignaturas del área de Organización de Empresas a lo largo de cinco cursos académicos. El análisis estadístico se realiza con el paquete estadístico IBM SPSS (versión 23).

Se registraron las notas obtenidas en uno de los exámenes de cada asignatura. Este examen, el primer parcial en cada caso de los dos que componían la evaluación de estas asignaturas, versaba sobre los contenidos relacionados con las principales funciones de la dirección de una empresa (planificación, organización, gestión de los recursos humanos y control). En todos los casos, uno de los grupos de alumnos (grupo experimental) había realizado en el aula 
previamente una actividad práctica en la que, exponiendo cómo la selección española de fútbol acaba ganando el Mundial de Suráfrica en 2010, se explica cuáles son y cómo se llevan a cabo las funciones de la dirección de cualquier empresa. Por su parte, el grupo control no realizaba esta actividad, sino que basaba el aprendizaje de dichos contenidos exclusivamente en la tradicional clase magistral, con una aproximación meramente teórica a las mencionadas funciones de la dirección de empresas apoyadas en ejemplos clásicos de índole meramente empresarial (en concreto, se presentaban casos referidos a un banco y a una empresa de automoción).

Se evaluó el rendimiento académico de los alumnos asignados a cada grupo (control y experimental) en cinco cursos (no todos consecutivos) y en cuatro asignaturas del área de Organización de Empresas. De un total de 1.546 estudiantes, 751 realizaron la práctica y 795 no la llevaron a cabo. Los datos se recogen comparativamente en cada caso por pares de grupos que realizaron el mismo examen el mismo día y a la misma hora. La asignación de los alumnos a un grupo u otro dependió del azar. Se eliminaron las notas de los no presentados al examen por no resultar significativas para el estudio.

Las universidades, los grados, las asignaturas y los cursos donde se llevó a cabo la recogida de las observaciones son los siguientes:

- Universidad de León (ULE), grado en Administración y Dirección de Empresas (GADE), Fundamentos de Administración de Empresas, Cursos 2012-2013 y 2013-2014.

- Universidad de León (ULE), grado en Ingeniería Informática (GII), Fundamentos de Administración de Empresas (FADE), Cursos 2013-2014 y 2014-2015.

- Universidad de Barcelona (UB), grado en Administración y Dirección de Empresas (GADE), Dirección Estratégica (DE), Cursos 2014-2015, 20162017 y 2017-2018.

- Universidad de Girona - Escuela Universitaria Mediterrani (UdG-Med), grado en Marketing (GM), Introducción a la Administración de Empresas (IAE), Cursos 2014-2015, 2016-2017 y 2017-2018.

- Universidad de Girona - Escuela Universitaria Mediterrani (UdG-Med), grado en Turismo (GT), Organización y Gestión de la Empresa Turística (OET), Cursos 2016-2017 y 2017-2018.

La práctica que llevaba a cabo el grupo experimental en el aula, de forma previa a la convocatoria de cada una de las pruebas de examen correspondientes, constaba de dos fases, tal y como las define la propuesta de GutiérrezAragón (2013). La primera consistía en el visionado del documental Informe Robinson: Cuando fuimos campeones (Canal Plus España, 2010), que narra la historia interna de la consecución del Mundial de Fútbol de Sudáfrica por parte de la Selección Española de Fútbol en 2010, contada por sus protagonistas y exponiendo detalles poco conocidos para la mayor parte de los alumnos, por lo que su visionado suele centrar su atención de principio a fin. A lo 
largo del relato se desgrana el papel que juegan cada uno de los miembros que participan en el proyecto (jugadores, cuerpo técnico, cuerpo médico, fisioterapeutas, cocineros, etc.) en la consecución final del objetivo perseguido.

La segunda fase consistía en la realización de una actividad en pequeños grupos en la que había que identificar, para el caso que acababan de visionar, los diferentes procesos dentro de cada una de las funciones de la dirección: planificación (qué, cómo, cuándo y quién llevó a cabo el proyecto de ganar ese Mundial de Fútbol), organización (funciones y tareas de cada miembro participante en el proyecto y estructura de la organización), gestión de los recursos humanos (selección, formación, motivación de los miembros del equipo) y control (toma de medidas preventivas antes del campeonato y aplicación de medidas correctivas en los periodos entre partidos y durante los partidos) (Gutiérrez-Aragón, 2013). Finalmente, el profesor explicaba su propia interpretación de las soluciones al caso propuesto. El objetivo final era establecer un modelo de aprendizaje activo y participativo que, basándose en un ejemplo real del mundo del deporte, hiciese comprender a los alumnos que el modo en el que se llevaron a cabo las funciones clásicas de la dirección condujeron al éxito final del proyecto, proporcionándoles así unas bases teóricas adecuadas para conocer las funciones de la dirección de empresas.

A los estudiantes del grupo control, por su parte, se les explicaban estos mismos contenidos basándose de forma exclusiva en la clase magistral. En ambos casos, los grupos experimental y control recibieron las mismas horas de docencia para explicar los referidos contenidos y en cada universidad y grado en los que se habían llevado a cabo la práctica el profesor que impartía las clases respectivas era el mismo.

Una vez que se realizaron los exámenes a cada grupo de alumnos (control y experimental) se procedió a la recogida y a la codificación de las calificaciones obtenidas. El estudio se encuadra en un diseño de intervención cuasiexperimental, ya que la asignación de los participantes por grupos no es aleatoria (Delgado, Llorca y Domènech, 2012). La manipulación de la variable independiente (grupo control o experimental) o de exposición es realizada por el experimentador. Se trata de un estudio de intervención de tipo transversal, ya que las mediciones se realizan en diversos grupos, pero una sola vez (crosssectional). Además, este estudio es de tipo retrospectivo: primero ocurre el efecto o la exposición y posteriormente se recogen los resultados (variable dependiente: nota).

\section{Resultados}

En la presente investigación se comparan los resultados académicos obtenidos en un examen entre dos grupos: control y experimental. Los resultados de este estudio ponen de manifiesto que las notas medias obtenidas por los alumnos del grupo experimental son superiores a las obtenidas por los alumnos del grupo control en todos los casos, tal y como se puede apreciar en la tabla 1. 
Tabla 1. Notas medias y desviaciones estándar para el grupo experimental y el grupo control en los diferentes cursos, asignaturas, grados y universidades

\begin{tabular}{|c|c|c|c|c|c|c|c|}
\hline Curso & Asignatura & Grado & Universidad & Grupo & $\mathbf{N}$ & Media & DE \\
\hline \multirow{2}{*}{$\begin{array}{l}2012- \\
2013\end{array}$} & \multirow{2}{*}{ FADE } & \multirow{2}{*}{ GADE } & \multirow{2}{*}{ ULE } & GC & 51 & 5,04 & 1,83 \\
\hline & & & & GE & 29 & 6,32 & 1,66 \\
\hline \multirow{2}{*}{$\begin{array}{l}2013- \\
2014\end{array}$} & \multirow{2}{*}{ FADE } & \multirow{2}{*}{ GADE } & \multirow{2}{*}{ ULE } & GC & 50 & 4,71 & 1,91 \\
\hline & & & & GE & 34 & 5,82 & 2,08 \\
\hline \multirow{2}{*}{$\begin{array}{l}2013- \\
2014\end{array}$} & \multirow{2}{*}{ FADE } & \multirow{2}{*}{ Gll } & \multirow{2}{*}{ ULE } & GC & 98 & 4,48 & 2,37 \\
\hline & & & & GE & 112 & 5,35 & 1,91 \\
\hline \multirow{2}{*}{$\begin{array}{l}2014- \\
2015\end{array}$} & \multirow{2}{*}{$\mathrm{DE}$} & \multirow{2}{*}{ GADE } & \multirow{2}{*}{ UB } & $\mathrm{GC}$ & 99 & 5,98 & 1,34 \\
\hline & & & & GE & 99 & 6,39 & 1,69 \\
\hline \multirow{2}{*}{$\begin{array}{l}2014- \\
2015\end{array}$} & \multirow{2}{*}{ IAE } & \multirow{2}{*}{ GM } & \multirow{2}{*}{ UdG-Med } & GC & 30 & 5,94 & 1,50 \\
\hline & & & & GE & 30 & 6,58 & 1,83 \\
\hline \multirow{2}{*}{$\begin{array}{l}2014- \\
2015\end{array}$} & \multirow{2}{*}{ FADE } & \multirow{2}{*}{ Gll } & \multirow{2}{*}{ ULE } & GC & 52 & 5,09 & 2,26 \\
\hline & & & & GE & 46 & 6,23 & 1,47 \\
\hline \multirow{2}{*}{$\begin{array}{l}2016- \\
2017\end{array}$} & \multirow{2}{*}{$\mathrm{DE}$} & \multirow{2}{*}{ GADE } & \multirow{2}{*}{ UB } & GC & 90 & 5,14 & 1,71 \\
\hline & & & & GE & 89 & 6,13 & 1,48 \\
\hline \multirow{2}{*}{$\begin{array}{l}2016- \\
2017\end{array}$} & \multirow{2}{*}{ IAE } & \multirow{2}{*}{ GM } & \multirow{2}{*}{ UdG-Med } & GC & 74 & 5,45 & 2,42 \\
\hline & & & & GE & 55 & 6,17 & 2,10 \\
\hline \multirow{2}{*}{$\begin{array}{l}2016- \\
2017\end{array}$} & & & & GC & 45 & 4,61 & 1,99 \\
\hline & OEI & GI & UdG-Med & GE & 62 & 6,24 & 1,95 \\
\hline $2017-$ & & & & GC & 92 & 5,02 & 1,48 \\
\hline 2018 & DE & GADE & UB & GE & 97 & 6,22 & 1,37 \\
\hline $2017-$ & & & & GC & 33 & 5,61 & 1,91 \\
\hline 2018 & IAE & GiM & UdG-Med & GE & 36 & 6,49 & 1,94 \\
\hline $2017-$ & OFT & GT & UdG-Med & GC & 81 & 4,80 & 1,92 \\
\hline 2018 & UEI & GI & UaG-IVIed & GE & 62 & 6,29 & 1,96 \\
\hline
\end{tabular}

$\mathrm{N}=$ número de estudiantes; $\mathrm{DE}$ = desviación estándar; $\mathrm{GE}=$ grupo experimental; $\mathrm{GC}$ = grupo control Fuente: elaboración propia a partir de datos recogidos por profesores de la Universidad de León (20122015), la Universitat de Barcelona (2014-2018) y la Escola Universitària Mediterrani, Universitat de Girona (2014-2018).

En la tabla 2 se presenta el análisis de las diferencias entre las notas medias de los grupos experimental y control obtenidas a partir de las observaciones en la Universidad de León. En las cuatro pruebas realizadas sobre grupos de alumnos de la ULE, las notas medias de los 251 alumnos de los grupos control oscilaron entre los 4,48 y los 5,09 puntos y el promedio de las calificaciones de los 221 alumnos de los grupos experimentales varió entre los 5,35 y los 6,32 puntos. La diferencia en puntos, siempre a favor del grupo experimental, osciló entre 0,87 y 1,28, lo que significó una desigualdad porcentual a favor que variaba entre un $19,42 \%$ y un $25,47 \%$. La diferencia total de media fue de 1,10 puntos a favor de los estudiantes del grupo experimental (diferencia porcentual media del 22,77\%).

Por lo que respecta a las tres pruebas llevadas a cabo sobre grupos de alumnos de la UB (ver tabla 3), las notas medias de los 281 alumnos de los grupos control oscilaron entre los 5,02 y los 5,98 puntos, y el promedio de las califi- 
Tabla 2. Notas medias y diferencias entre el grupo experimental y el grupo control (ULE)

\begin{tabular}{lccccc}
\hline & & $\begin{array}{c}\text { Año 1 } \\
\text { GADE, FADE }\end{array}$ & $\begin{array}{c}\text { Año 2 GADE, } \\
\text { FADE }\end{array}$ & $\begin{array}{c}\text { Año 2 } \\
\text { GII, FADE }\end{array}$ & $\begin{array}{c}\text { Año 3 } \\
\text { GII, FADE }\end{array}$ \\
\hline GC & Media & 5,04 & 4,71 & 4,48 & 5,09 \\
& $\mathrm{~N}$ & 51 & 50 & 98 & 52 \\
$\mathrm{GE}$ & Media & 6,32 & 5,82 & 5,35 & 6,23 \\
& $\mathrm{~N}$ & 29 & 34 & 112 & 46 \\
Total & $\mathrm{N}$ & 80 & 84 & 210 & 98 \\
Diferencia & & 1,28 & 1,11 & 0,87 & 1,14 \\
Diferencia porcentual & $25,40 \%$ & $23,57 \%$ & $19,42 \%$ & $22,40 \%$ \\
\hline
\end{tabular}

$\mathrm{N}=$ número de estudiantes; $\mathrm{GE}$ = grupo experimental; $\mathrm{GC}$ = grupo control

Fuente: elaboración propia a partir de datos recogidos por profesores de la Universidad de León (20122015).

Tabla 3. Notas medias y diferencias entre el grupo experimental y el grupo control (UB)

\begin{tabular}{|c|c|c|c|c|}
\hline & & $\begin{array}{c}\text { Año } 1 \\
\text { GADE, DE }\end{array}$ & $\begin{array}{c}\text { Año } 2 \\
\text { GADE, DE }\end{array}$ & $\begin{array}{c}\text { Año } 3 \\
\text { GADE, DE }\end{array}$ \\
\hline \multirow{2}{*}{ GC } & Media & 5,98 & 5,14 & 5,02 \\
\hline & $\mathrm{N}$ & 99 & 90 & 92 \\
\hline \multirow{2}{*}{ GE } & Media & 6,39 & 6,13 & 6,22 \\
\hline & $\mathrm{N}$ & 99 & 89 & 97 \\
\hline Total & $\mathrm{N}$ & 198 & 179 & 189 \\
\hline \multicolumn{2}{|c|}{ Diferencia } & 0,41 & 0,99 & 1,20 \\
\hline \multicolumn{2}{|c|}{ Diferencia porcentual } & $6,86 \%$ & $19,26 \%$ & $23,90 \%$ \\
\hline
\end{tabular}

$\mathrm{N}=$ número de estudiantes; $\mathrm{GE}$ = grupo experimental; $\mathrm{GC}$ = grupo control

Fuente: elaboración propia a partir de datos recogidos por profesores de la Universitat de Barcelona (20142018).

caciones de los 285 alumnos de los grupos experimentales varió entre los 6,22 y los 6,39 puntos. La diferencia en puntos a favor del grupo que sí realizó la actividad propuesta fluctuó entre 0,41 y 1,20 puntos, lo que significó una desigualdad porcentual a favor de entre un $6,86 \%$ y un $23,90 \%$. La diferencia total en media fue de 0,87 puntos a favor de los alumnos pertenecientes al grupo experimental (diferencia porcentual media del 16,11\%).

Por último, en las cinco pruebas realizadas sobre grupos de alumnos de la Escuela Mediterrani (UdG) (ver tabla 4), las notas medias de los 263 alumnos de los grupos control oscilaron entre los 4,61 y los 5,94 puntos, y el promedio de las calificaciones de los 265 alumnos de los grupos experimentales varió entre los 6,17 y los 6,58 puntos. La diferencia, siempre a favor del grupo experimental, osciló entre 0,64 y 1,63 puntos, lo que significó una diferencia porcentual que fluctuó entre un $10,77 \%$ y un $35,36 \%$. La diferencia total en media fue de 1,07 puntos a favor de los alumnos del grupo experimental (diferencia porcentual media del 20,30\%).

En definitiva, una vez analizados los datos obtenidos de los 1.546 alumnos (tabla 5), el resultado de la nota media de los 795 alumnos del grupo control de las tres universidades fue de 5,16 puntos, mientras que el promedio de las 
Tabla 4. Notas medias y diferencias entre el grupo experimental y el grupo control (Escuela Universitaria Mediterrani, UdG)

\begin{tabular}{lcccccc}
\hline & & $\begin{array}{c}\text { Año 1 } \\
\text { GM, IAE }\end{array}$ & $\begin{array}{c}\text { Año 2 } \\
\text { GM, IAE }\end{array}$ & $\begin{array}{c}\text { Año 3 GM, } \\
\text { IAE }\end{array}$ & $\begin{array}{c}\text { Año 1 GT, } \\
\text { OET }\end{array}$ & $\begin{array}{c}\text { Año 2 } \\
\text { GT, OET }\end{array}$ \\
\hline \multirow{2}{*}{ GC } & Media & 5,94 & 5,45 & 5,61 & 4,61 & 4,80 \\
& $\mathrm{~N}$ & 30 & 74 & 33 & 45 & 81 \\
$\mathrm{GE}$ & Media & 6,58 & 6,17 & 6,49 & 6,24 & 6,29 \\
Total & $\mathrm{N}$ & 30 & 55 & 36 & 62 & 62 \\
Diferencia & $\mathrm{N}$ & 60 & 129 & 69 & 107 & 143 \\
Diferencia porcentual & & 0,64 & 0,72 & 0,88 & 1,63 & 1,49 \\
\hline
\end{tabular}

$\mathrm{N}=$ número de estudiantes; $\mathrm{GE}=$ grupo experimental; $\mathrm{GC}=$ grupo control

Fuente: elaboración propia a partir de datos recogidos por profesores de la Escola Universitària Mediterrani, Universitat de Girona (2014-2018).

Tabla 5. Notas medias (aritméticas y ponderadas) y diferencias entre el grupo experimental y el grupo control registradas en el total de la muestra

\begin{tabular}{lccc}
\hline & Media & $\begin{array}{c}\text { TOTALES } \\
\text { (Medias aritméticas) }\end{array}$ & $\begin{array}{c}\text { TOTALES } \\
\text { (Medias ponderadas) }\end{array}$ \\
\hline GC & $\mathrm{N}$ & 5,16 & 5,13 \\
& Media & 795 & 795 \\
$\mathrm{GE}$ & $\mathrm{N}$ & 6,19 & 6,12 \\
Total & $\mathrm{N}$ & 751 & 751 \\
Diferencia & & 1.546 & 1.546 \\
\hline Diferencia porcentual & & 1,03 & 0,99 \\
\hline
\end{tabular}

$\mathrm{N}$ = número de estudiantes; $\mathrm{GE}$ = grupo experimental; $\mathrm{GC}$ = grupo control

Fuente: elaboración propia a partir de datos recogidos por profesores de la Universidad de León (20122015), la Universitat de Barcelona (2014-2018) y la Escola Universitària Mediterrani, Universitat de Girona (2014-2018).

calificaciones de los 751 alumnos del grupo experimental fue de 6,19 puntos. La diferencia a favor del grupo experimental fue, pues, de 1,03 puntos, lo que significó una desigualdad porcentual a favor del 19,98\%. Realizado el mismo análisis utilizando para su cálculo la técnica de medias ponderadas en función del número de alumnos que realizaba cada prueba (en vez de utilizar medias aritméticas para ello), se concluye que la nota media ponderada de los 795 alumnos del grupo control fue de 5,13 puntos, mientras que la media ponderada de las calificaciones de los 751 alumnos del grupo experimental fue de 6,12 puntos. La diferencia a favor del grupo que sí realizó la actividad propuesta fue, en función de esta forma de cálculo, de 0,99 puntos, lo que significó una desigualdad porcentual a favor de un 19,37\%. Los resultados obtenidos según se use una técnica u otra no parecen arrojar diferencias tan significativas como para considerar que el sesgo que pudiera derivarse de utilizar la primera de las técnicas sea lo suficientemente trascendente como para no recomendar su utilización en análisis de estas características. 
En cuanto a los resultados obtenidos con la prueba de comparación de grupos, t-test, se observa que, en la mayoría de los casos, en las doce observaciones de los distintos grupos (tabla 6) existen diferencias significativas a favor del grupo experimental. No obstante, las diferencias no son significativas en cuatro de los doce grupos. Concretamente se trataría de un grupo del grado en ADE de la UB y de los tres grupos de grado en Marketing de la Escuela Mediterrani (UdG).

Tabla 6. Resultados t-test de comparación de grupos (grupos control, sin práctica, o grupos experimentales, con práctica)

\begin{tabular}{lccc}
\hline \multicolumn{1}{c}{ Grupo } & $\boldsymbol{t}$ & $\boldsymbol{p}$ & IC (dif.) \\
\hline NGADEULE 1 & 3,10 & 0,003 & 0,46 a 2,1 \\
\hline NGADEULE 2 & 2,52 & 0,014 & 0,23 a 1,98 \\
\hline NGIIFULE 1 & 2,91 & 0,004 & 0,28 a 1,46 \\
\hline NGIIFULE 2 & 2,92 & 0,004 & 0,36 a 1,92 \\
\hline NGADEUB 1 & 1,92 & 0,056 & \\
\hline NGADEUB 2 & 4,13 & $<0,0001$ & 0,51 a 1,46 \\
NGADEUB 3 & 5,76 & $<0,0001$ & 0,79 a 1,61 \\
\hline NGMUdG 1 & 1,47 & 0,146 & \\
NGMUdG 2 & 1,76 & 0,081 & \\
NGMUdG 3 & 1,91 & 0,061 & \\
\hline NGTUdG 1 & 4,25 & $<0,0001$ & 0,87 a 2,40 \\
\hline NGTUdG 2 & 4,56 & $<0,0001$ & 0,85 a 2,14 \\
\hline
\end{tabular}

$t=$ valor de la prueba t-test; $p=$ grado de significación; IC (dif.): intervalo de confianza de la diferencia entre medias (grupos)

Fuente: elaboración propia a partir de datos recogidos por profesores de la Universidad de León (20122015), la Universitat de Barcelona (2014-2018) y la Escola Universitària Mediterrani, Universitat de Girona (2014-2018).

\section{Discusión y conclusiones}

La innovación docente como paradigma de pensamiento ha venido apostando en las últimas décadas de una forma clara por modelos educativos basados en el aprendizaje, en contraposición a los más tradicionales, apoyados principalmente en la clase magistral. La finalidad fundamental de dicho cambio es la de proporcionar a la sociedad actual universitarios con mayor autonomía y creatividad, así como una mejor preparación personal y profesional.

Bajo dichas premisas, el objetivo principal de este trabajo era evaluar la relación entre el desarrollo y la implantación de proyectos de innovación docente realizados en las aulas y el rendimiento académico del alumnado. Del análisis de los resultados obtenidos puede desprenderse que parece demostrarse que, al menos para el caso objeto del estudio, esta relación existe, ya que las notas medias de los grupos de alumnos del grupo experimental (compuesto por los estudiantes que llevaron a cabo la actividad práctica de innovación docente) siempre fueron más elevadas (unos en mayor intensidad y otros en menor intensidad) que las de los alumnos del grupo control (a quienes, no 
llevándola a cabo, se les impartieron los mismos contenidos mediante una docencia tradicional). En artículos académicos previos resulta posible comprobar que, cuando otros investigadores han realizado experiencias parecidas con dos muestras de estudiantes, una que conforma un grupo experimental al que se aplican novedades docentes y otra segunda que constituye un grupo control integrada por estudiantes que reciben una enseñanza bajo parámetros más tradicionales, se ha llegado a conclusiones similares en cuanto a la mejora del rendimiento académico (Jareño y López, 2015) o a la superior adquisición de competencias transversales o clave (Cárdenas, Pulido y Carrillo, 2016).

De todos modos, a pesar de que en la presente investigación han existido diferencias siempre a favor del grupo experimental, estas no son significativas en cuatro de los doce grupos. Es posible que esta circunstancia, al producirse sobre todo en los grupos de alumnos del grado en Marketing (UdG), sea debida a que precisamente estos estudiantes realizan en muchas asignaturas un gran número de actividades docentes innovadoras en la misma línea que la propuesta, siendo menos proclives a verse afectados por realizar una sola de estas actividades.

Esta experiencia no está exenta de limitaciones, como la posible falta de control sobre otras variables que, por desconocimiento, podrían haber influido en los resultados obtenidos por parte del alumnado (interacción de otras innovaciones docentes de otras asignaturas, existencia de capacidades diferentes en los integrantes de los grupos, etc.), o los potenciales sesgos que hayan podido introducir los propios profesores responsables de la experiencia. Por ello, en estudios posteriores se debería tratar de detectar y tener en consideración otras variables, como, por ejemplo, las que pueden haber influido en la reducción de los efectos de la innovación docente en los grupos del grado en Marketing antes mencionados. Por otro lado, a modo de indicador de satisfacción, hubiera sido interesante conocer y comparar entre los dos grupos (experimental y control) las valoraciones de los alumnos en las encuestas de evaluación docente, tanto de asignaturas como de profesores, pero lamentablemente ninguna de las universidades aporta las herramientas que hubieran permitido hacerlo discriminando ambos grupos.

En cualquier caso, el ensayo realizado pone en evidencia que en el entorno universitario y social actual, en concreto en el campo de asignaturas del área de Organización de Empresas, la propuesta de innovación educativa tiende a mejorar el rendimiento y el éxito académico. En el caso del presente estudio, el aprendizaje del modelo de organización propuesto, comprendiendo el importante papel de cada miembro que participó en el logro deportivo, supuso para los alumnos la posibilidad de asimilar conductas que conducen al éxito de cualquier proyecto empresarial. En definitiva, parece verificarse que utilizando estas herramientas los estudiantes logran un acercamiento más rápido y eficiente que por otras vías educativas más tradicionales a diversas nociones económico-empresariales, sobre todo aquellas relacionadas con el esfuerzo, el trabajo en equipo, la profesionalidad, la responsabilidad y la gestión de los recursos humanos y del talento (individual y colectivo). 


\section{Referencias bibliográficas}

Alliaud, A. (2015). Los artesanos de la enseñanza posmoderna: Hacia el esbozo de una propuesta para su formación. Historia y Memoria de la Educación, 1(1), 319-349.

Alonso, C.; Casablancas, S.; Martínez, S.; Sánchez, J.A. y Sancho, J.M. (2007). Relatos de innovación docente. Revista Latinoamericana de Tecnología Educativa, 6(2), 153-168.

Anderson, C. y Sally, D. (2013). The Numbers Game: Why everything you know about soccer is wrong. Nueva York: Penguin Group.

Arriba, R. de (2014). Seis razones para la innovación educativa en economía y una propuesta 2.0. Revista DIM: Didáctica, Innovación y Multimedia, 29, 1-10.

Bolívar, A.; Domingo, J. y Pérez-García, P. (2014). Crisis and Reconstruction of Teachers' Professional Identity: The Case of Secondary School Teachers in Spain. The Open Sports Sciences Journal, 7(Suppl-2, M4), 106-112.

Brunner, J.J.; Labraña, J.R.; Ganga, F. y Rodríguez-Ponce, E. (2019). Idea moderna de universidad: De la torre de marfil al capitalismo académico. Educación $X X 1,22(2), 119-140$. $<$ https://doi.org/10.5944/educxx1.22480>

Bustinza, Ó.F.; Tamayo, J.; Gutiérrez, L. y Orozco, L.M. (2010). Adaptación al EEES y experiencias de innovación docente en la asignatura de economía y organización de empresas impartida en la titulación de ITS de caminos, canales y puertos. En M. Pasadas-Fernández (coords.). Actas de las I Jornadas sobre Innovación Docente y Adaptación al EEES en las Titulaciones Técnicas (pp. 37-40). Granada: Godel Impresores Digitales.

Canal Plus España (2010). Informe Robinson: Cuando fuimos campeones [Documental]. Madrid: Sogecable. Recuperado de <https://vimeo.com/33289726>.

Cárdenas, P.J.; Pulido, J.I. y Carrillo, I. (2016). Adquisición de competencias en el Grado de Turismo mediante el aprendizaje basado en estudios de caso. Aula Abierta, 44(1), 15-22.

$<$ https://doi.org/10.1016/j.aula.2015.05.002>

Castañeda, L.; Esteve, F. y Adell, J. (2018). ¿Por qué es necesario repensar la competencia docente para el mundo digital? Revista de Educación a Distancia, 56, 1-20.

Correa, J.M.; Fernández, L.; Gutiérrez-Cabello, A.; Losada, D. y OchoaAizpurua, B. (2015). Formación del Profesorado, Tecnología Educativa e Identidad Docente Digital. RELATEC: Revista Latinoamericana de Tecnología Educativa, $14(1), 45-56$.

Cubeiro, J.C. y Gallardo, L. (2012). Por qué La Roja funciona y nuestro país (todavía) no. Barcelona: Alienta Editorial.

Delgado, M.; Llorca, J. y Domènech, J.M. (2012). Investigación científica: Fundamentos metodológicos y estadísticos. Barcelona: Signo.

Esteve, F. (2009). Bolonia y las TIC: De la docencia 1.0 al aprendizaje 2.0. La cuestión universitaria: Boletín de la Cátedra UNESCO de Gestión y Política Universitaria de la UPM, 5, 58-67.

Fernández-Ferrer, M. y Forés-Miravalles, A. (2018). Evaluación del desarrollo competencial en la educación superior: La perspectiva del profesorado universitario. Educar, 54(2), 391-410. <https://dx.doi.org/10.5565/rev/educar.799>

Fidalgo, A. (2011). La innovación docente y los estudiantes: La Cuestión Universitaria. Boletín de la Cátedra UNESCO de Gestión y Politica Universitaria de la UPM, 7, 84-91. 
Fonseca, M.C. y Rodríguez, L. (2009). Innovación docente y tasas de eficiencia. En J.D. Állvarez, N. Pellín y M.T. Tortosa-Ybáñez (coords.). VII Jornades de Xarxes d'Investigació en Docència Universitària: La qualitat del procés d'ensenyança I aprenentatge universitari des de la perspectiva del canvi (pp. 284-293). Alicante: Universidad de Alicante.

García, J.; Fernández-Gavira, J.; Sánchez, A.J. y Grimaldi, M. (2017). Gamificación y aplicaciones móviles para emprender: Una propuesta educativa en la enseñanza superior. International Journal of Educational Research and Innovation, 8, 233-259.

Goddard, J. y Dobson, S. (2011). The Economics of Football. 2. ${ }^{\mathrm{a}}$ ed. Cambridge: Cambridge University Press,

GonzÁlez, G. y Barba, J.J. (2014). Formación permanente y desarrollo de la identidad reflexiva del profesorado desde las perspectivas grupal e individual. Profesorado: Revista de Currículum y Formación del Profesorado, 18(3), 397-412.

- (2016). ¿Qué es la innovación docente?: Un cambio en las prácticas o de pensamiento docente. EmásF: Revista Digital de Educación Física, 38, 7-17.

Gutiérrez-Aragón, Ó. (2013). Fundamentos de Administración de Empresas. Madrid: Ediciones Pirámide.

Iglesias, J.; Toscano, M.O. y Román, M.C. (2012). Taller de prensa económica como instrumento de innovación educativa. Revista de Investigación Educativa, 30(2), 423-440.

Jano, D. y Ortiz, S. (2007). Experiencia de innovación docente en estadística económica. Revista de Docencia Universitaria, 2, 1-11.

JAREÑo, F. y López, R. (2015). Actividades de evaluación continua - correlación con la calificación de la prueba final y efecto sobre la calificación final: Evidencia en Administración y Dirección de Empresas. Revista Complutense de Educación, 26(2), 241-254. <http://dx.doi.org/10.5209/rev_RCED.2015.v26.n2.42948>

Jiménez, F. y Montijano, F. (2006). La economía experimental en las aulas. Iniciación a la Investigación, 2, 1-14.

Kuper, S. y Szymanski, S. (2012). Soccernomics: Why England loses, why Spain, Germany and Brazil win, and why the Us, Japan, Australia, Turkey-and even Iraq-are destined to become the kings of the world's most popular sport. 6. ${ }^{\mathrm{a}}$ ed. Nueva York: Nation Books.

LewIs, M. (2003). Moneyball: The art of winning an unfair game. Nueva York: W.W. Norton \& Company.

Méndez, T.J. (2018). La producción académica de los egresados del posgrado de instituciones de educación superior en México. Educar, 54(2), 261-282. $<$ https://dx.doi.org/10.5565/rev/educar.897>

Monreal, M.C. y Ruiz, E. (2010). La formación del profesorado en el marco de la innovación docente universitaria: El caso de la Universidad Pablo de Olavide. Revista de Docencia Universitaria, 4, 1-12.

Nash, J.F. (1950). Equilibrium points in n-person games. PNAS: Proceedings of the National Academy of Sciences of the United States of America, 36(1), 48-49.

Palacios, I. (2014a). Beautiful Game Theory: How soccer can help economics. Princeton: Princeton University Press.

- (2014b). Informe Robinson: El penalti de Nash. Recuperado de <http://cort.as/-JB-z>.

Pérez, J.V. (2014). Innovación docente. En E. Pastor y M.A. Martínez (coords.). Trabajo social en el siglo XxI: Una perspectiva internacional comparada (pp. 25-37). Madrid: Grupo 5. 
Perrenoud, P. (2001). Développer la pratique réflexive dans le métier d'enseignant: Professionnalisation et raison pédagogique. París: ESF Éditeur.

Peters, M.A. (2010). Re-Imagining the University in the Global Era. Policy Futures in Education, 8(2), 151-165.

Poblete, M.; Bezanilla, M.J.; Fernández, D. y Campo, L. (2016). Formación del docente en competencias genéricas: Un instrumento para su planificación y desarrollo. Educar, 52(1), 71-91. $<$ https://dx.doi.org/10.5565/rev/educar.713>

Salas, M.; Sánchez, M.T. y Rodríguez, N. (2012). Developing Generic Competences in the European Higher Education Area: A proposal for teaching the principles of economics. European Journal of Education, 47(2), 462-476. <https://doi.org/10.1111/j.1465-3435.2012.01525.x>

Sumpter, D. (2016). Soccermatics: Mathematical Adventures in the Beautiful Game. Londres: Bloomsbury Publishing.

Torrado, M. y Figuera, P. (2019). Estudio longitudinal del proceso de abandono y reingreso de estudiantes de Ciencias Sociales: El caso de Administración y Dirección de Empresas. Educar, 55(2), 71-91. <https://doi.org/10.5565/rev/educar.1022>

UNESCO (2018). Information and communication technology (ICT) in education. Recuperado de <http://cort.as/-JHjv>.

Viaña, E. (2013). Diez lecciones de gestión de «La Roja». Diario Expansión (18 de marzo). Recuperado de <http://cort.as/-KA7E >.

WaGner, T. (2012). Calling all Innovators. Educational Leadership, 69(7), 66-69. 